\title{
Asymptotics of the Dispersion Interaction: Analytic Benchmarks for van der Waals Energy Functionals
}

\author{
John F. Dobson, ${ }^{1,2,3}$ Angela White, ${ }^{1}$ and Angel Rubio ${ }^{4,3}$ \\ ${ }^{1}$ Nanoscale Science and Technology Centre, Griffith University, Nathan, Queensland 4111, Australia \\ ${ }^{2}$ LSI, Ecole Polytechnique, F91128 Palaiseau, France \\ ${ }^{3}$ Donostia International Physics Center (DIPC), E20018 Donostia/San Sebastián, Spain \\ ${ }^{4}$ European Theoretical Spectroscopy Facility (ETSF) and Departamento de Física de Materiales, \\ UPV/EHU and Unidad de Materiales Centro Mixto CSIC-UPV/EHU, E20018 San Sebastián, Spain
}

(Received 17 February 2005; published 22 February 2006)

\begin{abstract}
We show that the usual sum of $R^{-6}$ contributions from elements separated by distance $R$ can give qualitatively wrong results for the electromagnetically nonretarded van der Waals interaction between nonoverlapping bodies. This occurs for anisotropic nanostructures that have a zero electronic energy gap, such as metallic nanotubes or nanowires, and nanolayered systems including metals and graphene planes. In all these cases our analytic microscopic calculations give an interaction falling off with a power of separation different from the conventional value. We discuss implications for van der Waals energy functionals. The new nanotube interaction might be directly observable at submicron separations.
\end{abstract}

DOI: 10.1103/PhysRevLett.96.073201

Dispersion interactions [part of the van der Waals (vdW) energy] [1] are especially significant in soft matter. The vdW physics that we expose here could be relevant in predicting the energetics of bundles of metallic nanowires or nanotubes, layered metallic systems, $\pi$-conjugated systems including graphite, intercalated graphite, graphitic hydrogen storage systems and pi-stacked biomolecules, and other weakly bound ("soft") layered and striated nanosystems. Standard local (LDA) and gradient (GGA) density functionals for the electronic energy do not obtain any distant dispersion interaction, but density functionals have been derived recently that obtain, in a natural fashion, both distant dispersion interactions and their saturation at small distances. These and other numerically practicable vdW energy schemes available to date [1-8] for the above systems (in the electromagnetically nonretarded regime) have a "universal" feature: the distant vdW interaction energy between sufficiently separated subsystems is given qualitatively by a sum of contributions of form $R^{-6}$ between microscopic elements separated by distance $R$. This leads to "standard" power laws $E \propto-D^{-p}$ for the interaction energy between various macroscopic bodies separated by distance $D$ (column 3 of Table I). Although these universal asymptotic results are indeed valid for most macroscopic systems, we show below that they fail for the anisotropic nanostructures mentioned above. Column 2 of Table I summarizes the asymptotic $(D \rightarrow$ $\infty$ ) benchmarks that we propose below for the vdW energy of two parallel nanostructures of infinite extent.

To analyze these situations, we use the correlation energy $E_{c}^{\mathrm{RPA}}(D)$ from the random phase approximation (RPA) [12-17], a basic microscopic theory that does not rely on assumptions of locality, additivity, nor $R^{-6}$ contributions. Going beyond the RPA does not change the asymptotic power laws predicted here, unless the
PACS numbers: 34.20.Cf, 71.15.Mb, 73.22.-f, 81.05.Uw

exchange-correlation kernel $f_{\mathrm{xc}}$ [17-19] has a slower spatial decay than the bare Coulomb interaction, an unprecedented and unlikely scenario.

Where the separated subsystems exhibit lightly damped long-wavelength plasmons, we note [20] that the principal contribution to $E_{c}^{\mathrm{RPA}}(D)$ comes from the sum of coupledplasmon zero-point energies: otherwise we use the full RPA. Some essential common features of these systems will be abstracted from these specific calculations. We obtain analytic results for the asymptotic $(D \rightarrow \infty)$ regime in all cases, but in a later section we will also discuss systems near their equilibrium spacing.

Distant attraction between metallic linear structures. Consider two parallel, infinitely long conducting wires or tubes separated by a distance $D$ substantially exceeding their radius $b$, and with $b<\lambda$ where $\lambda$ is a bulk screening length. Both standard $\sum R^{-6}$ analysis [3] based on the vdW interaction between electrons localized in atoms or bonds, and recent functionals [21], give a vdW energy per unit length of the form $E \propto-D^{-5}$. Instead we consider the zero-point energy of the delocalized coupled one-

TABLE I. Asymptotic vdW energy of parallel structures. $K$ and $D_{0}$ are constants.

\begin{tabular}{lcc}
\hline \hline \multicolumn{1}{c}{ System } & Present & Standard \\
\hline 1D metals ${ }^{\mathrm{a}}$ & $-\mathbf{D}^{-\mathbf{2}}(\ln (\mathbf{K D}))^{-\mathbf{3} / \mathbf{2}}$ & $-D^{-5}$ \\
1D insulators [9] & $-D^{-5}$ & $-D^{-5}$ \\
2D metals [10,11] & $-\mathbf{D}^{-\mathbf{5} / \mathbf{2}}$ & $-D^{-4}$ \\
$\pi$-conjugated layers $^{\mathrm{a}}$ & $-\mathbf{D}^{-\mathbf{3}}$ & $-D^{-4}$ \\
1 metallic, 1 $\pi$ layer $^{\mathrm{a}}$ & $-\mathbf{D}^{-\mathbf{3}} \ln \left(\mathbf{D} / \mathbf{D}_{\mathbf{0}}\right)$ & $-D^{-4}$ \\
2D insulators [6] & $-D^{-4}$ & $-D^{-4}$ \\
Thick metals or ins. [11] & $-D^{-2}$ & $-D^{-2}$ \\
\hline \hline
\end{tabular}

$\mathrm{a} *$ Denotes new derivations given here. 
dimensional plasmon modes with wave number $q$ parallel to the long axis $[9,22]$. The radially smeared intrawire coulomb interaction is $w_{11}(q)=w_{22}(q)=-2 e^{2} \ln (q b)$ where 1 and 2 refer to the two wires, and we have assumed $q b \ll 1$, as appropriate when $D \gg b$. In the same limit the bare density-density response for electronic motion parallel to the wire is $\chi_{011}=\chi_{022}=N_{0} q^{2} /\left(m \omega^{2}\right)$ where $\omega$ is the frequency, $N_{0}$ is the number of electrons per unit length, and $m$ is the electron mass. RPA screening yields the interacting response of a single wire as $\chi_{11}=$ $\chi_{011} /\left(1-w_{11} \chi_{011}\right)$. The interwire Coulomb interaction in the present limit has a Bessel form, $w_{12}=2 e^{2} K_{0}(q D)$. The RPA equation for coupled 1D plasmons on two identical wires is $\chi_{11}^{2} w_{12}^{2}=1$, giving two roots for each $q$ : $\omega_{ \pm}(D)=c_{1 D}|q|\left(|\ln (q b)| \pm K_{0}(q D)\right)^{1 / 2}$. Here $c_{1 D}=$ $\left(2 N_{0} e^{2} / m\right)^{1 / 2}$ is a characteristic velocity. The vdW energy is the separation-dependent part of the sum of zero-point plasmon energies per unit length:

$$
\frac{E^{\mathrm{vdW}}}{L}=\frac{1}{2 \pi} \int_{-\infty}^{\infty} \frac{\hbar}{2}\left(\omega_{+}(D)+\omega_{-}(D)-2 \omega_{+}(\infty)\right) d q
$$

For $D \gg b$ we expanded to 2nd order in $K_{0}(q D) /|\ln (q b)|$ which is small near the peak of the integrand. This gave [9] approximately

$$
E^{\mathrm{vdW}} / L \approx-(16 \pi)^{-1} \hbar c_{1 D} D^{-2}(\ln (2.39 D / b))^{-3 / 2} .
$$

This approach is reasonable when the electron mean free path $d_{0}$ along the wire satisfies $d_{0}>D$. In fact bismuth nanowires [23] and conducting nanotubes [24] can both have $d_{0} \geq 1$ micron. Equation (1) differs from the widely accepted result $E \propto-D^{-5}$ by nearly three powers of $D$, and is necessarily dominant, at sufficiently large $D$, over any such higher-power contributions [arising from the remaining bound $\mathrm{sp}^{2}$ electrons (in nanotubes) and azimuthal $\pi$ plasmons]. Our plasmon model does give $D^{-5}$ if a pinning force is added to mimic an insulator [9].

Distant attraction between thin conducting layers. Consider infinite parallel metallic plates separated by distance $D$ and of thickness $b$, with $b \ll D, b<\lambda$ where $\lambda$ is the bulk screening length. As is already well known $[10,11]$, the zero-point energy of long-wavelength coupled 2D plasmons leads to an attraction of form $E \propto-D^{-5 / 2}$. The $\sum R^{-6}$ approach, correct for thin insulators, gives $E \propto$ $-D^{-4}$, different by 1.5 powers of $D$.

Distant attraction between planar $\pi$-conjugated systems. - What does the physics of long-wavelength excitations imply for the energetics of layered planar $\pi$-conjugated systems, such as the controversial [25-28] and technologically important [24,29] graphene-based systems? First, an isolated graphene layer at $T=0 \mathrm{~K}$ is not a metal but a zero-gap insulator [24]. Thus one cannot argue for a metallic $-D^{-5 / 2}$ energetics (as in the last paragraph) at large layer separation $D$ and $T=0 \mathrm{~K}$, even though band overlap makes graphite weakly metallic at the equilibrium layer spacing. We briefly derive below, however, our new result that the attractive energy between two well-separated graphene planes at $T=0 \mathrm{~K}$ is of form $-C_{3} D^{-3}$, closer to metallic $D^{-5 / 2}$ behavior than to insulating $D^{-4}$ behavior. All the new physics here comes from electrons close to the Fermi level: we can ignore the response of the tightly bound covalent $s p^{2}$ electrons, whose finite energy gap ensures that they produce a conventional vdW attraction of 2D insulator type (energy $\propto-D^{-4}$ ), negligible at large separations compared with the $D^{-3} \mathrm{vdW}$ attraction that we shall find between the $\pi_{z}$ electrons of interest here. The bonding and antibonding $\pi$ bands have a gapless band structure [24]. The energy near the $K$ points where the bands touch is given by $\varepsilon^{(1,2)}(\vec{p})=\mp \hbar v_{0}|\vec{p}|$ where $\vec{p}$ is the 2D crystal momentum measured from a $K$ point, and $v_{0}$ is a characteristic velocity (about $5.7 \times 10^{5} \mathrm{~m} / \mathrm{s}$ for graphene). From perturbation theory within a Wannier description [30,31], the zero-temperature density-density response $\chi_{\mathrm{KS}}$ of independent $\pi_{z}$ electrons moving in the groundstate Kohn-Sham (KS) potential of a gapless $\pi$ layer is then of the form $\chi_{\mathrm{KS}}\left(\vec{q}, \overrightarrow{0}, \overrightarrow{0}, z, z^{\prime}, \omega=i u\right)=$ $S(q, z) S\left(q, z^{\prime}\right)^{*} \bar{\chi}_{0}(\vec{q}, i u)$, with $\int S d z \rightarrow 1$ as $\vec{q} \rightarrow \overrightarrow{0}$. We found $[30,31]$ the effective $2 \mathrm{D}$ response $\bar{\chi}_{0}$ at small $q$ and imaginary frequency $\omega=i u$ to be

$$
\bar{\chi}_{0}(\vec{q}, i u) \approx-\left(2 \hbar v_{0}\right)^{-1} q\left(1+u^{2} /\left(v_{0} q\right)^{2}\right)^{-1 / 2},
$$

consistent with previous real- $\omega$ results [32]. Here we treat the response in each sheet as strictly two-dimensional, and ignore certain local-field effects, so that the only consequence of the periodic potential is to replace the 2D freeelectron bare response $-n_{0} q^{2} /\left(m u^{2}\right)$ by the zero-gap Bloch response (2). This is justified elsewhere [30,31]. We consider electron density perturbations of form $n_{1} \exp (i \vec{q} \cdot \vec{r}+u t)$ in layer $\# 1$, where $\vec{q}$ and $\vec{r}$ are two dimensional. Such charge disturbances interact via a Fourier transformed bare Coulomb potential,

$$
\begin{aligned}
& w_{11 \lambda}(q)=2 \pi \lambda e^{2} q^{-1}, \\
& w_{12 \lambda}(q)=2 \pi \lambda e^{2} q^{-1} \exp (-q D),
\end{aligned}
$$

for interactions within a layer and between two layers distant $D$, respectively. Then the RPA equation for the interacting density fluctuation in layer \#1 as driven by an external potential $v_{1}^{\operatorname{ext}} \exp (i \vec{q} \cdot \vec{r}+u t)$ is of timedependent mean-field form, $n_{1}=\bar{\chi}_{0}(q, i u) \times$ $\left(v_{1}^{\text {ext }}+w_{11} n_{1}\right)$. This applies in the absence of layer \#2 or equivalently for $D \rightarrow \infty$. Solving for $n_{1}$ we find a singlelayer density-density response

$$
\chi_{11 \lambda, D \rightarrow \infty} \equiv n_{1} / v_{1}^{\text {ext }}=\bar{\chi}_{0} /\left(1-w_{11 \lambda} \bar{\chi}_{0}\right) .
$$

With two layers present, the density response obeys coupled RPA equations $n_{1}=\chi_{11 \lambda, D \rightarrow \infty}\left(v_{1}^{\mathrm{ext}}+w_{12 \lambda} n_{2}\right)$, $n_{2}=\chi_{22 \lambda, D \rightarrow \infty}\left(v_{2}^{\text {ext }}+w_{21 \lambda} n_{1}\right)$. The solution is $\vec{n}=\chi \vec{v}^{\text {ext }}$ where $\vec{n}=\left(n_{1}, n_{2}\right)^{T}$ and similarly for $\vec{v}^{\text {ext }}$, while the components of the $2 \times 2$ matrix $\chi$ are $\chi_{11 \lambda, D}=\chi_{11 \lambda, D \rightarrow \infty} /(1-$ $\left.w_{12 \lambda} \chi_{11 \lambda, D \rightarrow \infty}\right)$ and $\chi_{12 \lambda, D}=w_{12 \lambda} \chi_{11 \lambda, D \rightarrow \infty} \chi_{11 \lambda, D}$. For the 
case of two identical layers considered here, the other elements are $\chi_{22 \lambda, D}=\chi_{11 \lambda, D}$ and $\chi_{21 \lambda, D}=\chi_{12 \lambda, D}$.

In the present system the response (4) of a single layer, continued to the real frequency axis, yields no lightly damped plasmons (poles) for small $q$, so that a sum of plasmon zero-point energies cannot be used to evaluate the $\mathrm{vdW}$ interaction. Instead we consider the electromagnetically nonretarded groundstate electronic correlation energy, which for a general inhomogeneous electronic system is given exactly by the adiabatic connection fluctuation-dissipation theorem (see, e.g., [20]):

$$
E_{c}=-\frac{\hbar}{2 \pi} \int_{0}^{1} d \lambda \int d \vec{r} d \vec{r}^{\prime} \frac{e^{2}}{\left|\vec{r}-\vec{r}^{\prime}\right|} \int_{0}^{\infty} \Delta \chi_{\lambda}\left(\vec{r}, \vec{r}^{\prime}, i u\right) d u
$$

Here $\Delta \chi_{\lambda}=\chi_{\lambda}-\chi_{0}$, where $\chi_{\lambda}$ is the electron densitydensity response function at reduced Coulomb interaction $\lambda e^{2} /\left|\vec{r}-\vec{r}^{\prime}\right|$. Applying (5) to the present layer geometry and Fourier transforming parallel to the layers we find that the separation-dependent part $E^{\mathrm{vdW}} / A$ of the energy per unit area is

$$
\frac{E_{c}(D)-E_{c}(\infty)}{A}=-\frac{\hbar}{\pi} \int_{0}^{\infty} d u \int_{0}^{1} \frac{d \lambda}{\lambda} \int_{0}^{\infty} \frac{2 \pi q d q}{(2 \pi)^{2}}\left(w_{11 \lambda}\left(\chi_{11 \lambda D}-\chi_{11 \lambda, D \rightarrow \infty}\right)+w_{12 \lambda} \chi_{12 \lambda D}\right) .
$$

Within the RPA approximation, Eqs. (2) and (3), plus (4) and the equations following it, show that each term of form $w \chi$ in (6) depends on $u$ solely through the dimensionless combination $x=u /\left(v_{0} q\right)$. The remaining dependence of $w \chi$ on $q$ is solely via $y=q D$. Thus (6) has a scaling form

$$
\begin{aligned}
E^{\mathrm{vdW}} / A & =\hbar \int_{0}^{1} \frac{d \lambda}{\lambda} \int_{0}^{\infty} q d q \int_{0}^{\infty} d u G\left(\lambda, \frac{u}{v_{0} q}, q D\right) \\
& =\frac{\hbar v_{0}}{D^{3}} \int_{0}^{1} \frac{d \lambda}{\lambda} \int_{0}^{\infty} y^{2} d y \int_{0}^{\infty} d x G(\lambda, x, y),
\end{aligned}
$$

where $G(\lambda, x, y)$ is independent of $D$. We numerically evaluated the dimensionless 3D integral in (7) for graphene parameters [30,31], giving the interaction energy per unit area in Gaussian esu units:

$$
\begin{aligned}
E^{\mathrm{vdW}} / A & =-7.745_{7} \times 10^{-2} \hbar v_{0} D^{-3} \\
& =-2.003_{6} \times 10^{-2} e^{2} D^{-3} .
\end{aligned}
$$

This $D^{-3}$ form shows that the gapless $\pi$-conjugated planes behave in this respect more like metals $(E \propto$ $\left.-D^{-5 / 2}\right)$ than insulators $\left(E \propto-D^{-4}\right)$, despite the lack of undamped 2D plasmon modes on a single $\pi$ sheet.

Parallel metallic and $\pi$-conjugated planes. - Another interesting case is the interaction between a $\pi$-conjugated layer and a metallic 2D layer with Fermi energy $\varepsilon_{F}$ (e.g., an undoped and a doped graphene sheet). For $D \gg D_{0}=$ $\hbar^{2} v_{0}^{2} /\left(2 \pi e^{2} \varepsilon_{F}\right)\left[=O(1 \mathrm{~nm})\right.$ for $\varepsilon_{F}=O(0.02 \mathrm{eV})$ as in a bulk graphite layer] the methods described above give an energy per unit area [c.f. (8)]

$$
E^{\mathrm{vdW}} / A \approx-C e^{2} D^{-3} \ln \left(D / D_{0}\right), \quad(C \text { const }) .
$$

As in the case of two nonmetallic gapless $\pi$ layers, the result (9) disagrees with standard theories.

Interaction energy near overlap. - We now discuss possible difficulties with the nonasymptotic, near-equilibrium energetics of the present systems, especially graphenes. The commonest $a b$ initio approach, the LDA, misses distant dispersion interactions entirely [33], and yet gives a good lattice spacing [34] and good breathing phonon frequencies [35] in graphite, (unlike GGAs [4,36]). Recent experiments [25,26], however, lead one to suspect [28] that the LDA pays for its neglect of dispersion physics by severely underestimating the equilibrium binding energy of graphite. LDA also underbinds related fullerene systems [27]. This phenomenon has been investigated in layered jellium analogs, via fully nonlocal many-body correlation theory ([37], Fig. 4 of [17]). It was found that either layerlayer forces or binding energy have serious errors near equilibrium, when distant dispersion forces are underestimated. The underestimation is related in turn to the lack of distant correlated fluctuations, especially those oriented parallel to the layers. Thus these low- $q$ fluctuations can have effects even near the equilibrium spacing. The addition of explicit $R^{-6} \mathrm{vdW}$ terms has been a common remedy for stretched graphitic systems [3,28], and recently several seamless vdW schemes have been proposed $[6,7,16]$, based on approximations for response functions. References [6,7] are the most practical, and are qualitatively successful in graphitics. [7] overestimates the binding energy of small systems but correspondingly obtains a large binding energy of two graphene layers (more than twice that from [6] or from LDA, and consistent with experiment). Ideally a single theory should give reliable results for small and extended systems. Could it be that the key is a correct treatment of the fluctuations parallel to a long axis in the extended cases, the same fluctuations responsible for the unusual asymptotics exposed here that is absent in [6,7]? These fluctuations are of course dominant only at large separations but they might not be negligible near the equilibrium spacing, where all wavelengths can contribute. We speculate further that the same physics might apply in other large finite $\pi$-conjugated systems (e.g., planar melanin layers, carotenes, fullerenes [27]) where, as the system size increases, the electronic gap diminishes while longerwavelength excitations become possible.

Summary and Discussion. - Our new results [see (1), (8), and (9) and Table I] show that usual sum of $C_{6} R^{-6}$ terms incorrectly predicts the dependence of the dispersion energy on separation $D$ for a range of systems. Simple energy functionals presently available all have standard $\sum C_{6} R^{-6}$ asymptotics. A finite sum of multipole, or triplet 
and higher terms will also not reproduce what we have discussed. The standard asymptotics fails when the component systems (i) are metallic (or have a zero electronic Bloch bandgap), and (ii) are spatially extended in at least one dimension, so that long-wavelength (low- $q$ ) charge fluctuations can occur, and (iii) are of nanoscopic dimensions in another spatial direction, so that the electronelectron screening is reduced compared with $3 \mathrm{D}$ bulk metallic systems, leaving a divergent screened polarizability at low frequency and wave number. [Thick metal slabs, for example, violate (iii): they have complete screening and exhibit a conventional power law, $E \propto-D^{-2}$. See, e.g., [11]]. Where free low- $q$ plasmons exist, conditions (i)-(iii) imply that they will be gapless. The same conditions ensure that the usual spatially local approximation for the dielectric function [2] is invalid. Our results provide unequivocal asymptotic benchmarks that are not satisfied by the existing simplified van der Waals energy formulas, because they do not treat in enough detail the fluctuations along the extended space dimension. In the last section we have further motivated the possibility that the same fluctuation physics may be relevant in the systems considered here, even near their equilibrium spacing. Investigation of this question requires a seamless energy formalism that is fully nonlocal-e.g., RPA-like theories $[11,16,17,20]$. Such calculations are only now becoming possible for 3D systems [38,39], with no converged results available to date for the present zero-gap cases. Simplified vdW energy functionals are therefore certainly needed for routine modeling, and the above considerations suggest that existing functionals may need further refinement to take explicit account of large-scale geometry and/or nonlocal entities such as electronic bandgap [20]. We note finally that our work predicts novel differences in the forces between conducting and nonconducting nanotubes or wires, that might be directly measurable for low-index nanotubes at submicron separations [9], and that could even affect selfassembly processes. These considerations might also affect the analysis of some seminal experiments $[25,26]$ concerning graphitic cohesion, because these relied at some point on theory involving a sum of $R^{-6}$ contributions.

We thank I. D'Amico, P. Garcia-Gonzalez, J. Jung, L. Reining, E. Gray, and P. Meredith for discussions. J.F.D. acknowledges support from Australian Research Council Grant No. DP0343926, UPV/EHU, Ecole Polytechnique and CNRS, and the hospitality of A.R. and Dr. L. Reining. A. R. was supported by the Network of Excellence NANOQUANTA (NMP4-CT-2004500198), and the Humbolt Foundation.

[1] J. Mahanty and B. Ninham, Dispersion Forces (Academic, London, 1976); D. Langbein, Theory of van der Waals Attraction (Springer-Verlag, Berlin, 1974).

[2] E. M. Lifshitz, Sov. Phys. JETP 2, 73 (1956).
[3] L. A. Girifalco, M. Hodak, and R. S. Lee, Phys. Rev. B 62, 13104 (2000).

[4] H. Rydberg et al., Surf. Sci. 532, 606 (2003).

[5] H. Rydberg, B. I. Lundqvist, D. C. Langreth, and M. Dion, Phys. Rev. B 62, 6997 (2000).

[6] H. Rydberg et al., Phys. Rev. Lett. 91, 126402 (2003).

[7] M. Dion et al., Phys. Rev. Lett. 92, 246401 (2004).

[8] A. D. Mackerell, J. Phys. Chem. B 102, 3586 (1998).

[9] J.F. Dobson and A. White (unpublished).

[10] M. Bostrom and B.E. Sernelius, Phys. Rev. B 61, 2204 (2000).

[11] J. F. Dobson et al., Australian J. Chem. 54, 513 (2001).

[12] J.F. Dobson, in Topics in Condensed Matter Physics, edited by M.P. Das (Nova, New York, 1994), Chap. 7; see also, cond-mat 0311371.

[13] J. M. Pitarke and A. G. Eguiluz, Phys. Rev. B 57, 6329 (1998).

[14] F. Furche, Phys. Rev. B 64, 195120 (2001).

[15] M. Fuchs and X. Gonze, Phys. Rev. B 65, 235109 (2002).

[16] J.F. Dobson and J. Wang, Phys. Rev. Lett. 82, 2123 (1999).

[17] J. Jung, P. Garcia-Gonzalez, J.F. Dobson, and R. W. Godby, Phys. Rev. B 70, 205107 (2004).

[18] E. K. U. Gross and W. Kohn, Phys. Rev. Lett. 55, 2850 (1985).

[19] J. F. Dobson and J. Wang, Phys. Rev. B 62, 10038 (2000).

[20] J. F. Dobson et al., Int. J. Quantum Chem. 101, 579 (2005).

[21] J. Kleis, P. Hyldgaard, and E. Schröder, Comput. Mater. Sci. 33, 192 (2005).

[22] P. Longe and S. Bose, Phys. Rev. B 48, 18239 (1993).

[23] A. D. Grozav and E. Condrea, J. Phys. Condens. Matter 16, 6507 (2004).

[24] R. Saito, G. Dresselhaus, and M. Dresselhaus, Physical Properties of Carbon Nanotubes (Imperial College, London, 1998).

[25] L. X. Benedict et al., Chem. Phys. Lett. 286, 490 (1998).

[26] R. Zacharia, H. Ulbricht, and T. Hertel, Phys. Rev. B 69, 155406 (2004).

[27] F. Tournus, J.-C. Charlier, and P. Melinon, J. Chem. Phys. 122, 094315 (2005).

[28] M. Hasegawa and K. Nishidate, Phys. Rev. B 70, 205431 (2004).

[29] V. V. Simonyan and J. K. Johnson, J. Alloys Compd. 330332, 659 (2002).

[30] J.F. Dobson and A. Rubio, cond-mat/0502422.

[31] J.F. Dobson (unpublished).

[32] J. Gonzalez, F. Guinea, and M. A. H. Vozmediano, Phys. Rev. B 63, 134421 (2001).

[33] W. Kohn, Y. Meir, and D. E. Makarov, Phys. Rev. Lett. 80, 4153 (1998).

[34] J.-C. Charlier, X. Gonze, and J.-P. Michenaud, Phys. Rev. B 43, 4579 (1991).

[35] L. Wirtz and A. Rubio, Solid State Commun. 131, 141 (2004).

[36] Z. Yan, J. P. Perdew, and S. Kurth, Phys. Rev. B 61, 16430 (2000).

[37] J. F. Dobson and J. Wang, Phys. Rev. B 69, 235104 (2004).

[38] T. Miyake et al., Phys. Rev. B 66, 245103 (2002).

[39] A. Marini, P. Garcia-Gonzalez, and A. Rubio, cond-mat/ 0510221. 